mit

$$
\psi(x)=\sum_{k} \psi(k) u_{k}(x)
$$

(wenn die Voraussetzungen (38) und (39) gemacht werden). Nun gilt für alle $t$

$$
\frac{\partial}{\partial x_{\nu}(1)} \widetilde{S}_{+}^{\prime}(1,2)=-\frac{\partial}{\partial x_{\nu}(2)} \widetilde{S}_{+}^{\prime}(1,2),
$$

wenn man (21) benutzt (die bei $t_{1}=t_{2}$ auftretenden Singularitäten heben sich gerade heraus). Die $u_{i}$ haben die Form $e^{i\left(\mathfrak{p}_{i} \mathfrak{r}-\omega_{i} t\right)}$. Behandeln wir der Einfachheit halber die $\left(\mathfrak{p}_{i}, \omega_{i}\right)$ als diskret, so wird wegen (A 4)

$$
\widetilde{S}_{+}^{\prime}=\Sigma f\left(\mathfrak{p}_{i}, \omega_{i}\right) e^{i\left[\mathfrak{p}_{i}\left(\mathfrak{r}_{1}-\mathrm{r}_{2}\right)-\omega_{i}\left(t_{1}-t_{2}\right)\right]}
$$

sein. Dies ${\widetilde{S_{+}}}^{\prime}(1,2)$ wird aus dem durch (A 1$)$ bestimmten $\widetilde{S}_{+}(1,2)$ durch Einschalten der Wechselwirkung hervorgehen. Daraus folgt unmittelbar die Gleichung (43).

$$
\begin{aligned}
& \text { Nach (43), (A } 2 \text { ) und (A 3) ist } \\
& {\widetilde{S_{+}}}_{t_{1} \rightarrow-\infty}(1,2)=-\sum \tilde{u}_{i}\left(\mathfrak{r}_{1},-\infty\right) \tilde{u}_{i} *\left(\mathfrak{r}_{2}, t_{2}\right) \\
& =-\sum u_{k}\left(\mathrm{r}_{1},-\infty\right)(U(0,2) \psi(2) U(2,-\infty) \Phi, \\
& U(0,-\infty) \psi(k) \Phi) .
\end{aligned}
$$

Daher ist

$$
\widetilde{u}_{k}^{*}(2)=\left(\psi(2) \Phi, \Phi_{-k}\right)
$$$$
\text { mit } \Phi_{-k}=U(0,-\infty) \psi(k) \Phi
$$

eine mögliche Darstellung; $\boldsymbol{\Phi}_{-k}$ ist der Zustand, in dem das $k$-Elektron vernichtet ist. Ferner gilt

$i \frac{\partial}{\partial t} \tilde{u}_{k}^{*}(x)=E \tilde{u}^{*}(x)-\left(E-\widetilde{\omega}_{k}\right) u_{k}^{*}(x)$

$$
\text { d. h. } \quad \tilde{u}_{k}(x)=e^{i \tilde{\omega}_{k} t} \tilde{u}_{k}(\mathfrak{r}) \text {. }
$$

$\widetilde{\omega}_{k}$ gibt den Energiebeitrag des $k$-Elektrons an. Bei dieser Überlegung ist die sog. Møllersche Relation

$$
U(0,-\infty) H_{0}(-\infty)=H(0) U(0,-\infty)
$$

benutzt (s. z. B. Glaser und Zimmermann ${ }^{8}$ ), die wiederum nur im Teilraum der Zustände freier Teilchen als gültig angesehen werden kann. Als Gesamtenergie folgt sofort (44).

\section{Darstellung von $\Phi$ aus $\varphi$}

Man kann für $A$ und $\psi$ bekanntlich Integralgleichungen (Yang und Feldman ${ }^{18}$ ) aufstellen, bei denen die Operatoren $A_{\text {in }}$ und $\psi_{\text {in }}$ durch

$$
\begin{aligned}
\Psi(\mathfrak{r},-\infty)=\psi_{\text {in }}(\mathfrak{r},-\infty), \\
\boldsymbol{A}(\mathfrak{r},-\infty)=A_{\text {in }}(\mathfrak{r},-\infty)
\end{aligned}
$$

definiert sind und den wechselwirkungsfreien Operatorengleichungen genügen. Sie hängen mit den $\psi$ der Wechselwirkungsdarstellung durch

$$
\psi(x)=U^{*}(0,-\infty) \psi_{\text {in }}(x) U(0,-\infty)
$$

zusammen. Hieraus folgt

$$
\psi_{\text {in }}(x) \boldsymbol{\Omega}=0 .
$$

Aus den $\psi_{\text {in }} *(k)$ (Erzeugungsoperatoren) mit

$$
\psi_{\text {in }}(x)=\Sigma \psi_{\text {in }}(k) u_{k}(x)
$$

kann man nach der Becker-Leibfried-Methode den Hilbert-Raum der Streuzustände aufbauen (der mit dem durch die $\psi(k)$ erzeugten unitär äquivalent ist). Betrachten wir nur diesen, so ist klar, daß aus

$$
\varphi_{t_{i} \rightarrow-\infty}(|1 \ldots N|)=\left(\psi_{\text {in }} *(1) \ldots \psi_{\text {in }}{ }^{*}(N) \mathbf{Q}, \Phi\right)
$$

alle Komponenten von $\boldsymbol{\Phi}$ gewonnen werden können. Denn $\Phi$ sollte nach Voraussetzung ein Zustand aus $N$ Elektronen sein, und ein solcher hat nur Projektionen in den Raum

$$
\begin{aligned}
& \int \mathrm{d} \mathfrak{r}_{1} \ldots d r_{N} f(1 \ldots N) \psi_{\text {in }} *(1) \ldots \psi_{\text {in }} *(N) \mathbf{Q} . \\
& 18 \text { C. N. Yang u. D. Feldman, Phys. Rev. } \mathbf{7 9} \text {, } \\
& 972 \text { [1950]. }
\end{aligned}
$$

\title{
Stationäre magneto-hydrodynamische Stoßwellen beliebiger Stärke
}

\author{
Von R. LÜST \\ Aus dem Max-Planck-Institut für Physik, Göttingen \\ (Z. Naturforschg. 10a, 125-135 [1955]; eingegangen am 24. Dezember 1954)
}

\begin{abstract}
Es werden für stationäre magneto-hydrodynamische Stoßwellen das Dichte- und Druckverhältnis, die Richtungsänderung des Magnetfeldes und die Geschwindigkeit hinter der Front in Abhängigkeit von der Machschen Zahl bestimmt. Es zeigt sich, daß drei verschiedene Arten von Stoßwellen in einem Plasma mit Magnetfeld existieren können, die im Grenzübergang zu unendlich schwachen Stößen in Schallwellen bzw. Alfvénsche Wellen übergehen. Die aufgestellten Gleichungen werden für ein einatomiges Gas und für verschiedene Stärken und Richtungen des Magnetfeldes als Funktion der Machschen Zahl numerisch gelöst und diskutiert.
\end{abstract}

$\mathrm{I}^{\mathrm{n}}$ n einer früheren Arbeit ${ }^{1}$ waren die Grundgleichungen für magneto-hydrodynamische Stoßwellen in einem Plasma mit unendlicher Leitfähig-

${ }^{1}$ R. Lüst, Z. Naturforschg. 8a, 277 [1953]. Im folgenden mit I bezeichnet. S. auch $\mathrm{F}$. de Hoffmann u. E. Teller, Phys. Rev. 80, 692 [1950]. keit abgeleitet worden. Wenn man sich auf eine makroskopische Beschreibung solcher Stoßwellen beschränkt, d. h. sich nur für die Dichte, den Druck, die Temperatur, das Magnetfeld usw. vor und hinter der Stoßfront interessiert, so zeigen alle diese Größen beim Durchgang durch die Stoß- 
front eine sprunghafte Änderung, die von der Geschwindigkeit der Stoßfront abhängt. Für die normalen hydrodynamischen Stoßwellen lassen sich für diese Dichte-, Druck- und Temperatursprünge explizite Gleichungen angeben ${ }^{2}$. Im folgenden sollen analoge Gleichungen für Stoßwellen im Magnetfeld aus den Grundgleichungen abgeleitet werden und damit die Verhältnisse bei verschiedenen Stoßstärken näher untersucht werden. Es wird sich zeigen, daß z. B. das Dichteverhältnis im allgemeinen Fall durch eine Gleichung 3. Grades bestimmt ist. Eine Untersuchung ähnlicher Art ist auch von $\mathrm{Helfer}{ }^{3}$ angestellt worden.

Für das folgende sollen dieselben Bedingungen und Bezeichnungen wie in I zugrunde gelegt werden. Die Leitfähigkeit sei also stets unendlich groß, die Stoßfront eben und unendlich ausgedehnt, sowie stationär. Sie liege in der $y z$-Ebene, und die Geschwindigkeit $U$ der Stoßfront verlaufe stets in der $x$-Richtung. Weiterhin sei die Unabhängigkeit aller Größen von der $z$-Koordinate vorausgesetzt. Schließlich sei angenommen, daß die Stoßwelle in ein ruhendes Gas hineinlaufe, d. h. daß die Geschwindigkeit $\mathfrak{u}_{1}$ der Massenteilchen vor der Front (gemessen im gleichen Koordinatensystem wie $U$ ) Null sei. Wenn mit $\mathfrak{b}$ die Relativgeschwindigkeit zur Stoßfront bezeichnet wird, so gilt [vgl. Gl. (I, $30)]$ :

$$
\begin{aligned}
& v_{x 1}=-U ; v_{y 1}=0 ; \\
& v_{x 2}=u_{x 2}-U ; v_{y 2}=u_{y 2} .
\end{aligned}
$$

(Der Index , , "“ bezieht sich stets auf Werte vor der Front, der Index ,,2“ auf solche hinter der Front.)

Abb. 1. Stoßfront (in der $y z$-Ebene liegend und sich nach rechts bewegend) und Magnetfeld für $M_{+1}>1$.

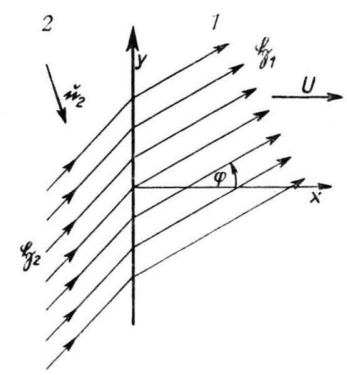

Das Magnetfeld $\mathfrak{\mathfrak { H }}$ liege in der $x y$-Ebene irgendwie schief zur Stoßfront; der Winkel zwischen Magnetfeld und $x$-Richtung heiße $\varphi$ (s. Abb. 1).

2 S. z. B. K. Oswatitsch, Gasdynamik, S. 94 , Springer-Verlag, Wien 1952.

${ }^{3}$ H. L. Helfer, Astrophys. J. 117, 177 [1953].

\section{Ausbreitungsgeschwindigkeiten von Wellen unendlich kleiner Amplitude im Magnetfeld}

Aus der normalen Gasdynamik weiß man, daß Stoßwellen nur möglich sind, wenn die Geschwindigkeit $U$ größer als die Geschwindigkeit $c_{\mathrm{s}}$ ist, mit der sich Wellen unendlich kleiner Amplitude - das sind Schallwellen - im Gas ausbreiten können. Das Verhältnis $M=U / c_{\mathrm{s}}$ bezeichnet man als Machsche Zahl, und Stoßwellen können also nur bei $M>1$ existieren.

Ebenso bekommt man auch nur magneto-hydrodynamische Stoßwellen, falls die Frontgeschwindigkeit $U$ größer ist als die Geschwindigkeit, mit der sich eine unendlich kleine Störung im Magnetfeld fortpflanzt. Die Geschwindigkeit solcher „Schallwellen“ in einem Plasma hängt von der Stärke des Magnetfeldes und von der Fortpflanzungsrichtung (relativ zum Magnetfeld) ab. Außerdem existieren im Magnetfeld sowohl transversale als auch longitudinale Wellen. Dies ist zuerst von Herlofson ${ }^{4}$ und von van de Hulst ${ }^{5}$ gezeigt worden.

Die Geschwindigkeiten solcher Schallwellen seien hier noch einmal angegeben, da sie für das folgende wichtig sind. In I ergab sich durch Grenzübergang zu unendlich schwachen Stoßwellen für das Quadrat der Ausbreitungsgeschwindigkeit $c$ in der $x$-Richtung folgende quadratische Gleichung [s. Gl. $(\mathrm{I}, 65)]$ :

$$
c^{4}-\left(c_{\mathrm{s}}^{2}+\frac{1}{4 \pi \varrho} H^{2}\right) c^{2}+\frac{1}{4 \pi \varrho} H_{x}^{2} c_{\mathrm{s}}^{2}=0 .
$$

Hierin ist $\varrho=$ Dichte, $H^{2}=H_{x}{ }^{2}+H_{y}{ }^{2}, c_{\mathrm{s}}{ }^{2}=\mathrm{d} p / \mathrm{d} \varrho$ $=\gamma p / \varrho=$ Schallgeschwindigkeit ohne Magnetfeld, $p=$ Druck und $\gamma=$ Verhältnis der spezifischen Wärmen. Aus Gl. (2) erhält man die beiden Lösungen

$$
\begin{aligned}
c_{+}{ }^{2}= & \frac{1}{2}\left(c_{\mathrm{s}}{ }^{2}+\frac{1}{4 \pi \varrho} H^{2}\right) \\
& +\frac{1}{2} \sqrt{\left(c_{\mathrm{s}}{ }^{2}+\frac{1}{4 \pi \varrho} H^{2}\right)^{2}-\frac{1}{\pi \varrho} H_{x}{ }^{2} c_{\mathrm{s}}{ }^{2}}
\end{aligned}
$$

und

$$
\begin{aligned}
c_{-}{ }^{2}= & \frac{1}{2}\left(c_{\mathrm{s}}{ }^{2}+\frac{1}{4 \pi \varrho} H^{2}\right) \\
& -\frac{1}{2} \sqrt{\left(c_{\mathrm{s}}{ }^{2}+\frac{1}{4 \pi \varrho} H^{2}\right)^{2}-\frac{1}{\pi \varrho} H_{x}{ }^{2} c_{\mathrm{s}}{ }^{2} .}
\end{aligned}
$$

${ }^{4}$ N. Herlofson, Nature, Lond. 165, 1020 [1950].

5 H. C. van de Hulst, Problems of Cosmical Aerodynamics, p. 45 (Dayton, Ohio, Central Air Documents Office 1951). 
Im Grenzfall $\varphi=0$, d. h. das Magnetfeld liegt parallel zur Ausbreitungsrichtung, wird daraus:

$$
c_{+}{ }^{2}=c_{\mathrm{s}}{ }^{2} \text { und } c_{-}{ }^{2}=\frac{1}{4 \pi \varrho} H_{x}{ }^{2}=c_{\mathrm{A}}{ }^{2} .
$$

In diesem Fall hat man einmal die normale longitudinale Schallwelle mit der Geschwindigkeit $c_{\mathrm{s}}$ und außerdem die transversale Alfvénsche Welle mit der Geschwindigkeit $c_{\mathbf{A}}$. Die normale Schallwelle wird durch das Magnetfeld nicht beeinflußt, da die Schwingungsrichtung longitudinal und damit parallel zum Magnetfeld ist. Das Magnetfeld liefert aber eine Quersteifigkeit (Torsionssteifigkeit) analog wie in einem festen Körper und ermöglicht so auch die Ausbreitung transversaler Wellen. In einem festen Körper ist die Geschwindigkeit der transversalen Wellen stets kleiner als die der longitudinalen. Hier dagegen kann die Geschwindigkeit der transversalen Wellen auch größer sein als die der longitudinalen, falls das Magnetfeld genügend stark ist.

Im anderen Grenzfall $\varphi=\pi / 2$, d. h. das Magnetfeld liegt senkrecht zur Ausbreitungsrichtung, bekommt man:

$$
c_{+}{ }^{2}=c_{\mathrm{s}}{ }^{2}+\frac{1}{4 \pi \varrho} H_{y}{ }^{2}=c^{* 2} \text { und } c_{-}{ }^{2}=0 .
$$

Hier hat man nur eine mögliche Welle, nämlich eine longitudinale Welle mit der Geschwindigkeit $c^{*}$, während eine transversale Welle in der $x$ Richtung nicht existieren kann, da das Magnetfeld in diesem Fall keine Quersteifigkeit in bezug auf die $x$-Richtung liefert. Für die longitudinale Welle wirkt sich das Magnetfeld so aus, daß die Kompressibilität herabgesetzt wird. Daher ist die Fortpflanzungsgeschwindigkeit $c^{*}$ größer als $c_{\mathrm{s}}$ (s. auch Abschn. 3).

Im allgemeinsten Fall $(\varphi \neq 0$ und $\varphi \neq \pi / 2)$ sind die Verhältnisse analog wie in einem festen anisotropen Körper. Durch das Magnetfeld tritt eine Kopplung der longitudinalen und transversalen Wellen ein. Die beiden möglichen Wellen mit den Geschwindigkeiten $c_{+}$bzw. $c_{-}$sind weder rein longitudinal noch rein transversal, sondern die Schwingungsrichtung liegt schief zur Fortpflanzungsrichtung und ist abhängig von der Größe der jeweiligen Fortpflanzungsgeschwindigkeit. Nach van de Hulst ist der Winkel $\alpha$ der Schwingungsrichtung mit der $x$-Achse gegeben durch:

$$
\operatorname{tg} \alpha_{ \pm}=\frac{c_{\mathrm{s}}{ }^{2}-c_{ \pm}^{2}}{c_{ \pm}^{2}} \operatorname{ctg} \varphi \text {. }
$$

Für die Fortpflanzungsgeschwindigkeiten gilt in jedem Fall, daß $c_{+}>c_{-}$ist. Ist das Magnetfeld nur sehr schwach, so bekommt man

$$
c_{+}{ }^{2} \rightarrow c_{\mathrm{s}}^{2} \text { mit } \alpha_{+} \rightarrow 0
$$

und

$$
c_{-}{ }^{2} \rightarrow c_{\mathrm{A}^{2}}{ }^{2} \text { mit } \alpha_{-} \rightarrow \pi / 2 .
$$

Dann sind also die Wellen wie im Fall $\varphi=0$ rein longitudinal und rein transversal. Wenn das Magnetfeld dagegen sehr stark ist, so hat man folgende Grenzwerte:

$$
c_{+}{ }^{2} \rightarrow c_{\mathrm{A}}^{2} / \cos ^{2} \varphi \text { mit } \alpha_{+} \rightarrow \pi / 2+\varphi
$$

und

$$
c_{-}^{2} \rightarrow c_{\mathrm{s}}^{2} \cos ^{2} \varphi \text { mit } \alpha_{-} \rightarrow \varphi .
$$

Die Schwingungsrichtung der Welle mit der Geschwindigkeit $c_{+}$liegt also hier senkrecht zum Magnetfeld und die der Welle mit der Geschwindigkeit $c_{-}$parallel zum Magnetfeld.

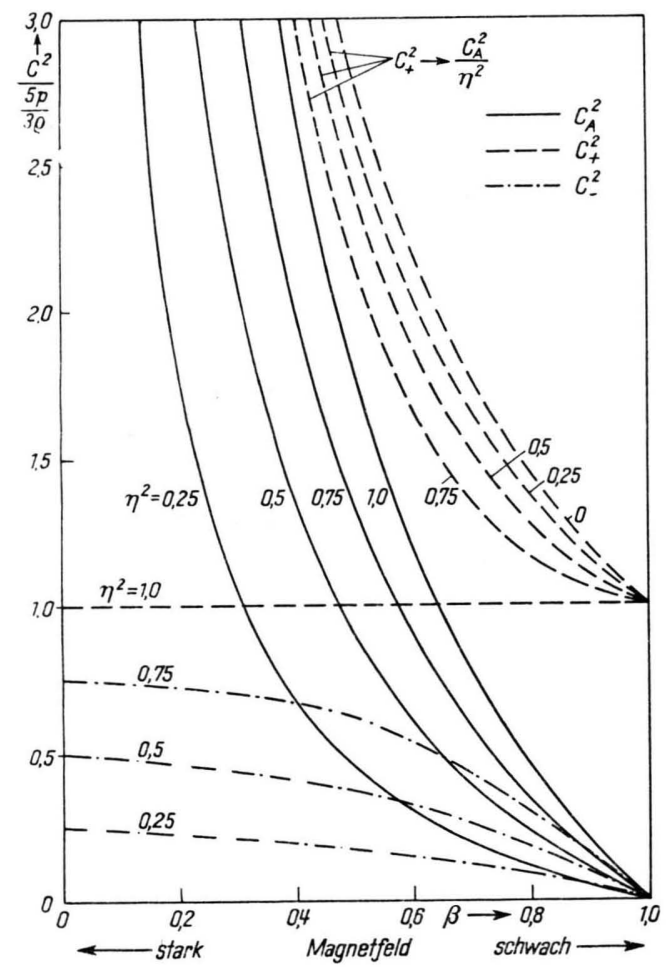

Abb. 2. Die ,Schallgeschwindigkeiten“ $c_{+}, c_{\mathrm{A}}$ und $c_{-}$ in Abhängigkeit von der Stärke des Magnetfeldes in verschiedenen Richtungen zum Magnetfeld, bezogen jeweils auf die Schallgeschwindigkeit ohne Magnetfeld.

Der Vollständigkeit halber sei bemerkt, daß noch eine dritte rein transversale Welle existiert (sofern $\varphi \neq \pi / 2$ ), deren Schwingungsrichtung senkrecht auf der $x y$-Ebene steht. Dies ist eine Alf- 
vénsche Welle mit der Fortpflanzungsgeschwindigkeit

$$
c_{\mathrm{A}}{ }^{2}=\frac{1}{4 \pi \varrho} H_{x}^{2} .
$$

Diese ist nicht gekoppelt mit den beiden Wellen, deren Schwingungsrichtungen in der $x y$-Ebene liegen. Da hier nur Vorgänge in der $x y$-Ebene betrachtet werden sollen, interessiert diese Wellenart im Grunde für das folgende nicht.

In Abb. 2 sind die drei verschiedenen Fortpflanzungsgeschwindigkeiten der Wellen mit unendlich kleiner Amplitude aufgetragen in Abhängigkeit von der Stärke des Magnetfeldes für ein einatomiges Gas $(\gamma=5 / 3)$. Die verschiedenen Geschwindigkeiten sind jeweils bezogen auf die Schallgeschwindigkeit $c_{\mathrm{s}}$ ohne Magnetfeld. Der Kurvenparameter ist $\eta^{2}=\cos ^{2} \varphi$. Der Faktor $\beta$ ist das Verhältnis der inneren Energie des Gases zur gesamten inneren Energie [s. Abschn. 3, Gl. (23)]. In Abb. 3 sind die verschiedenen Schallgeschwindigkeiten noch einmal in einem Polardiagramm bei festgehaltener Richtung des Magnetfeldes aufgetragen. Die Kurven geben also an, bis wohin beispielsweise kleine Störungen in einer Sekunde gelaufen sind.

\section{Parallele Stoßwellen}

Wie schon in I gezeigt wurde, hat das Magnetfeld keinen Einfluß, wenn die Fortpflanzung der Stoßwelle parallel zum Magnetfeld erfolgt $(\varphi=0)$. Die entsprechenden Grundgleichungen sind z. B. in I, Gl. (41 a-d), angegeben worden. Daraus lassen sich das Dichte-, das Druckverhältnis usw. in Abhängigkeit von der Machschen Zahl $M_{1}=U / c_{\mathrm{s} 1}$ berechnen ${ }^{2}$.

Diese seien hier noch einmal zum Vergleich mit den folgenden angegeben:

$$
\begin{aligned}
\frac{\varrho_{2}}{\varrho_{1}} & =\frac{M_{1}{ }^{2}}{\mu^{2} M_{1}{ }^{2}+\left(1-\mu^{2}\right)}, \\
\frac{p_{2}}{p_{1}} & =\left(1+\mu^{2}\right) M_{1}{ }^{2}-\mu^{2}, \\
\frac{u_{x 2}}{c_{\mathrm{s}_{1}}} & =\left(1-\mu^{2}\right) M_{1}\left(1-\frac{1}{M_{1}{ }^{2}}\right) .
\end{aligned}
$$

Hierin ist $\mu^{2}=(\gamma-1) /(\gamma+1)$

(d. h. $\mu^{2}=1 / 4$, wenn $\gamma=5 / 3$ ).

Man sieht aus Gl. (9), daß das Dichteverhältnis bei sehr starken Stößen $\left(M_{1} \rightarrow \infty\right)$ gegen den Grenzwert $1 / \mu^{2}$ geht und das Druckverhältnis gegen Unendlich.

\section{Senkrechte Stoßwellen}

Wenn die Ausbreitung der Stoßwellen senkrecht zum Magnetfeld erfolgt $(\varphi=\pi / 2)$, so kann man sehr leicht von den normalen hydrodynamischen Stoßgleichungen zu denen im Magnetfeld übergehen, indem man an Stelle des Gasdruckes den Gesamtdruck

$$
p^{*}=p+p_{\mathrm{m}}=p+\frac{1}{8 \pi} \mathfrak{\mathfrak { G }}^{2},
$$

und an Stelle der inneren spezifischen Energie des Gases die gesamte innere Energie

$$
e^{*}=e+e_{\mathrm{m}}=e+\frac{1}{8 \pi \varrho} \mathfrak{S g}^{2}
$$

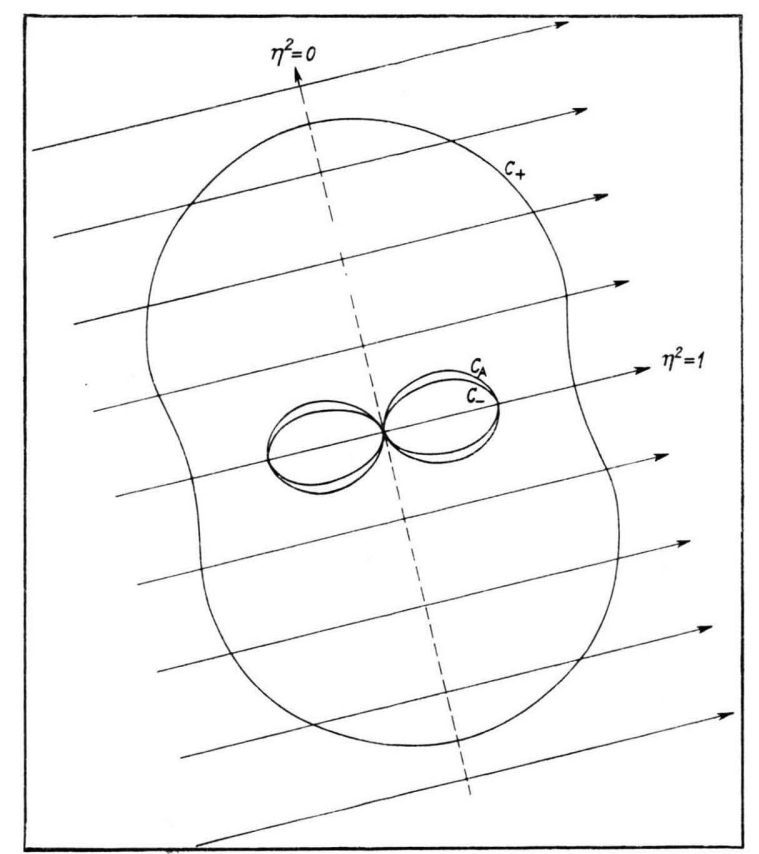

Abb. 3. Polardiagramm für die ,Schallgeschwindigkeiten" $c_{+}, c_{\mathrm{A}}$ und $c_{-}$bei $\beta=0,75$.

in die Gleichungen einsetzt [s. I, Gln. (40a) und $(40 \mathrm{~b})]$. Im folgenden soll in mehr qualitativer Weise der Einfluß des Magnetfeldes abgeschätzt werden, während die genauere Ableitung der Gleichungen für die Dichte-, Druckverhältnisse usw. im nächsten Abschnitt bei den schiefen Stoßwellen miteingeschlossen ist. Diese Betrachtung kann sich auch für andere Probleme, bei denen Magnetfelder zugegen sind, als nützlich erweisen; (z. B. kann das von Chandrasekhar ${ }^{6}$ abgeleitete Virialtheorem

${ }^{6}$ S. Chandrasekhar u. E. Fermi, Astrophysic. J. 118, 116 [1953]. 
im zweidimensionalen Fall damit auf einfache Weise gewonnen werden).

Da auf der Vorder- und Rückseite der Stoßfront die Entropie stets als konstant angenommen wird (Reibung und Wärmeleitung werden vernachlässigt), gilt für die Vorder- bzw. Rückseite der Front folgende Beziehung zwischen Gasdruck und Gasdichte:

$$
p=A \varrho^{\prime},
$$

wobei $A$ eine Konstante ist. Der magnetische Druck $p_{\mathrm{m}}$ ist gegeben durch

$$
p_{\mathrm{m}}=\frac{1}{8 \pi} H_{y}{ }^{2} \quad\left(\mathfrak{S}^{2}=H_{y}{ }^{2}\right) .
$$

Aus I, Gln. (45 c) und (45d), folgt $H / \varrho=$ const. (16)

Diese Gleichung drückt die Tatsache aus, daß wegen der vorausgesetzten unendlichen Leitfähigkeit die Materie streng an das Magnetfeld gekoppelt ist. Mit Hilfe von Gl. (15) und (16) bekommt man dann

$$
p_{\mathrm{m}}=B \varrho^{1 / \mathrm{m}},
$$

wobei $\gamma_{\mathrm{m}}=2$ und $B$ eine weitere Konstante ist. Man sieht also, daß man den Zusammenhang zwischen dem magnetischen Druck und der Gasdichte mit einem ,,Verhältnis der spezifischen Wärmen" $\gamma_{\mathrm{m}}=2$ beschreiben kann. Mit diesem Wert kann man jetzt analog wie bei einem idealen Gas die innere Energie des Magnetfeldes ableiten. Für ein ideales Gas gilt:

$$
e=\frac{1}{\gamma-1} \frac{p}{\varrho} .
$$

Für die magnetische Energie ergibt sich dann:

$$
e_{\mathrm{m}}=\frac{1}{\gamma_{\mathrm{m}}-1} \frac{p_{\mathrm{m}}}{\varrho}=\frac{1}{8 \pi \varrho} H_{y}{ }^{2} .
$$

Das gleiche gilt für die Schallgeschwindigkeit im Magnetfeld [s. Gl. (5)] :

$$
c^{* 2}=c_{\mathrm{s}}^{2}+c_{\mathrm{m}}^{2}=\gamma \frac{p}{\varrho}+\gamma_{\mathrm{m}} \frac{p_{\mathrm{m}}}{\varrho} .
$$

Wenn man nun für die gesamte Energie einführt:

$$
e^{*}=\frac{1}{\gamma^{*}-1} \frac{p^{*}}{\varrho},
$$

so kann man damit ein effektives $\gamma^{*}$ definieren in analoger Weise, wie man das für die Materie und Strahlung getan hat? ${ }^{7}$ (Es sei bemerkt, daß auch andere Definitionen von $\gamma^{*}$ möglich sind.) Aus

7 S. Chandrasekhar, An Introduction to the Study of Stellar Structure, p. $55 \mathrm{ff}$., Unit. of Chicago Press 1939.
Gl. (21) erhält man zusammen mit Gl. (18) und (19):

$$
\gamma^{*}=\frac{\gamma e+\gamma_{\mathrm{m}} e_{\mathrm{m}}}{c^{*}} .
$$

Als Maß für die Stärke des Magnetfeldes sei das Verhältnis $\beta$ zwischen der inneren Energie des Gases und der gesamten inneren Energie eingeführt, also

$$
e=\beta e^{*} ; \quad e_{\mathrm{m}}=(1-\beta) e^{*} \quad(0 \leq \beta \leq 1) .
$$

Dann wird aus Gl. (22)

$$
\gamma^{*}=\beta \gamma+(1-\beta) \gamma_{\mathrm{m}} \text {. }
$$

$\gamma^{*}$ wird für ein einatomiges Gas zwischen $5 / 3$ und 2 liegen. Ein größeres $\gamma^{*}$ bedeutet, daß die Materie inkompressibler und dementsprechend die Schallgeschwindigkeit senkrecht zum Magnetfeld vergrößert wird. Für die Stoßwellen heißt dies, daß der Dichte- und Druckanstieg bei Gegenwart eines Magnetfeldes zunächst nicht so groß ist wie ohne Magnetfeld. $\gamma^{*}$ bleibt aber beim Durchgang einer Stoßwelle nicht konstant, da $\beta$ nicht konstant bleibt. Für sehr starke Stöße nimmt das Druckverhältnis sehr stark zu, während das Dichteverhältnis beschränkt bleibt [s. Gl. (9) und (10)]. Die innere Energie des Gases $e_{2}$ kann so mit zunehmender Stoßstärke beliebig zunehmen, dagegen bleibt die innere Energie des Magnetfeldes $e_{\mathrm{m}_{2}}$ stets endlich. Der Faktor $\beta_{2}$ auf der Rückseite der Front geht also mit wachsender Stoßstärke gegen 1, und damit wird $\gamma_{2}{ }^{*}$ den Grenzwert $\gamma$ haben. Das Dichteverhältnis wird demnach für sehr starke Stoßwellen den gleichen Grenzwert haben wie bei den normalen hydrodynamischen Stoßwellen, nämlich $(\gamma+1) /(\gamma-1)$. Man sieht so, daß für sehr starke senkrechte Stoßwellen das Magnetfeld praktisch keine Rolle mehr spielt. Es wird sich zeigen, daß dies auch für Stoßwellen gilt, die sich nicht senkrecht zum Magnetfeld fortpflanzen.

\section{Schiefe Stoßwellen}

Das Magnetfeld soll nun eine beliebige Richtung zur Stoßfront haben, also $0<\varphi \leq \pi / 2$. Der Gesamtdruck in der $x$-Richtung $p_{x}{ }^{*}$ ist dann nach Gl. (I, 35) gegeben durch

$$
\begin{aligned}
p_{x}{ }^{*} & =p+\frac{1}{8 \pi}\left(H_{y}{ }^{2}-H_{x}{ }^{2}\right) \\
& =p+\frac{1}{8 \pi}\left(1-2 \eta^{2}\right) H^{2},
\end{aligned}
$$

worin

$$
\eta=\cos \varphi
$$


ist. Für die beiden Schallgeschwindigkeiten in der $x$-Richtung bekommt man aus Gl. $(3 \mathrm{a})$ und $(3 \mathrm{~b})$ mit Hilfe von Gl. (23) und Gl. (26)

wobei

$$
c_{ \pm}^{2}=\frac{1}{2 \mu^{2}\left(1-\mu^{2}\right) \beta} \frac{p}{\varrho} K_{ \pm},
$$

$$
\begin{aligned}
K_{ \pm}= & \mu^{2}\left(1+\mu^{2}\right) \beta+\left(1-\mu^{2}\right)^{2}(1-\beta) \\
& \pm\left\{\left[\mu^{2}\left(1+\mu^{2}\right) \beta+\left(1-\mu^{2}\right)^{2}(1-\beta)\right]^{2}\right. \\
& \left.-4\left(1+\mu^{2}\right)\left(1-\mu^{2}\right)^{2} \beta(1-\beta) \eta^{2}\right\}^{1 / 2} .
\end{aligned}
$$

a) Änderung des Magnetfeldes

Aus Gl. (I, 61) bekommt man:

$$
\begin{aligned}
& H_{y 2}\left\{\frac{1}{4 \pi}\left(H_{x 2}\right)^{2}-\varrho_{2}\left(v_{x 2}\right)^{2}\right\} \\
& =H_{y 1}\left\{\frac{1}{4 \pi}\left(H_{x 1}\right)^{2}-\varrho_{1}\left(v_{x 1}\right)^{2}\right\} .
\end{aligned}
$$

Es sei zunächst vorausgesetzt, daß

$$
\left(v_{x 2}\right)^{2} \neq \frac{1}{4 \pi \varrho_{2}}\left(H_{x 2}\right)^{2}
$$

sei. Dann ist das Verhältnis der $y$-Komponenten des Magnetfeldes gegeben durch:

$$
\begin{aligned}
\frac{H_{y 2}}{H_{y 1}} & =\frac{\varrho_{1}\left(v_{x 1}\right)^{2}-\frac{1}{4 \pi}\left(H_{x 1}\right)^{2}}{\varrho_{2}\left(v_{x 2}\right)^{2}-\frac{1}{4 \pi}\left(H_{x 2}\right)^{2}} \\
& =y C,
\end{aligned}
$$

wobei $y=\varrho_{2} / \varrho_{1}$ und

$$
C=\frac{1-b \varrho_{1}}{1-b \varrho_{2}},
$$

während $b$ eine Konstante ist:

$$
b=\frac{\left(H_{x}\right)^{2}}{4 \pi \varrho^{2}\left(v_{x}\right)^{2}},
$$

da nach Gl. (I, 52 e und 52e)

$$
\varrho_{1} v_{x 1}=\varrho_{2} v_{x 2} \text { und } H_{x 1}=H_{x 2}
$$

ist. Die letzte Gleichung und Gl. (31) beschreiben also das Magnetfeld auf der Rückseite der Stoßfront in Abhängigkeit von dem Magnetfeld der Vorderseite, sofern das Dichteverhältnis $y$ bekannt ist.

\section{b) Druckverhältnis}

Aus Gl. (I, 59) erhält man mit der Enthalpie für ein ideales Gas

$$
i=\frac{1+\mu^{2}}{2 \mu^{2}} \frac{p}{\varrho}
$$

für das Dichteverhältnis

$$
y=\frac{\varrho_{2}}{\varrho_{1}}=\frac{p_{2}+\mu^{2}\left[p_{1}+\frac{1}{8 \pi}\left(\Delta H_{y}\right)^{2}\right]}{p_{1}+\mu^{2}\left[p_{2}+\frac{1}{8 \pi}\left(\Delta H_{y}\right)^{2}\right]} .
$$

Eine Umformung liefert mit $\tau=1 / \underline{Q}$

$$
-\frac{\Delta p}{\Delta \tau}=\frac{p_{2}+\mu^{2}\left[p_{1}+\frac{1}{8 \pi}\left(\Delta H_{y}\right)^{2}\right]}{\left(1-\mu^{2}\right) \tau_{1}} .
$$

Aus dem Impulssatz für die $x$-Komponente Gl. (I, 52, bl) bekommt man

$-\frac{\Delta p}{\Delta \tau}=\left(\varrho_{1} v_{x 1}\right)^{2}+\frac{1}{8 \pi} \frac{1}{\Delta \tau}\left\{\left(H_{y_{2}}\right)^{2}-\left(H_{y_{1}}\right)^{2}\right\}$,

so daß sich schließlich für den Druck auf der Rückseite der Front ergibt:

$p_{2}=\left(1-\mu^{2}\right) \varrho_{1}\left(v_{x 1}\right)^{2}-\mu^{2} p_{1}$

$-\frac{1}{8 \pi}\left[\mu^{2}\left(\Delta H_{y}\right)^{2}-\frac{\tau_{1}}{\Delta \tau}\left(1-\mu^{2}\right)\left\{\left(H_{y_{2}}\right)^{2}-\left(H_{y_{1}}\right)^{2}\right\}\right]$.

Benutzt man Gl. (31), so wird daraus

$$
\begin{aligned}
p_{2}= & \left(1-\mu^{2}\right) \varrho_{1}\left(v_{x 1}\right)^{2}-\mu^{2} p_{1}-\frac{1}{8 \pi}\left[\mu^{2}(y C-1)^{2}\right. \\
& \left.-\left(1-\mu^{2}\right) y \frac{1}{1-b \varrho_{2}}(y C+1)\right]\left(H_{y 1}\right)^{2} .
\end{aligned}
$$

Führt man jetzt die Machsche Zahl

$M_{1}{ }^{2}=\frac{\left(u_{x 1}\right)^{2}}{c_{1}{ }^{2}}=\frac{U^{2}}{c_{1}{ }^{2}} \quad\left(c_{1}=c_{+1}\right.$ bzw. $\left.=c_{-1}\right)$

ein und wendet die Gln. (23), (26) und (27) an, so erhält man schließlich für das Druckverhältnis in Abhängigkeit von der Machschen Zahl

$$
\begin{aligned}
\frac{p_{2}}{p_{1}}= & \frac{1}{2 \mu^{2} \beta_{1}} K_{1} M_{1}^{2}-\mu^{2} \\
& -\frac{1}{2 \mu^{2} \beta_{1}}\left(1-\mu^{2}\right)\left(1-\beta_{1}\right)\left(1-\eta_{1}{ }^{2}\right) \\
& \cdot\left[\mu^{2}(y C-1)^{2}+\left(1-\mu^{2}\right) y D(y C+1)\right] .
\end{aligned}
$$

Hierin ist

$C=\frac{K_{1} M_{1}^{2}-2\left(1-\mu^{2}\right)^{2}\left(1-\beta_{1}\right) \eta_{1}^{2}}{K_{1} M_{1}^{2}-2\left(1-\mu^{2}\right)^{2}\left(1-\beta_{1}\right) \eta_{1}^{2} y}$,

$D=\frac{1}{1-b \varrho_{2}}=\frac{K_{1} M_{1}{ }^{2}}{K_{1} M_{1}{ }^{2}-2\left(1-\mu^{2}\right)^{2}\left(1-\beta_{1}\right) \eta_{1}{ }^{2} y}$

und $K_{1}=K_{+1}$ bzw. $=K_{-1}$.

\section{c) Dichteverhältnis}

In Gl. (38) stehen bis auf das Dichteverhältnis $y$ nur Größen von der Vorderseite der Front. Das Dichteverhältnis ist durch Gl. (33) gegeben. Setzt man in diese die Gl. (38) ein, so ergibt sich:

$$
y=\frac{K_{1} M_{1}{ }^{2}-\left(1-\mu^{2}\right)^{2}\left(1-\eta_{1}{ }^{2}\right) y D(y C+1)}{\mu^{2}\left[K_{1} M_{1}{ }^{2}+2 \beta_{1}\left(1-\mu^{4}\right)\right]+\mu^{2}(1-\mu)^{2}\left(1-\beta_{1}\right)\left(1-\eta_{1}{ }^{2}\right)\left[(y C-1)^{2}-y D(y C+1)\right]} .
$$


Daraus bekommt man eine Gleichung 3. Grades für das Dichteverhältnis $y$ :

mit den Koeffizienten

$$
a_{3} y^{3}+a_{2} y^{2}+a_{1} y+a_{0}=0
$$

$a_{3}=4 \mu^{2}\left(1-\mu^{2}\right)^{4}\left(1-\beta_{1}\right)^{2} \eta_{1}^{2}\left\{K_{1} M_{1}^{2}+2\left(1-\mu^{4}\right) \beta_{1} \eta_{1}^{2}\right\}$,

$a_{2}=\left(1-\mu^{2}\right)\left(1-\beta_{1}\right) K_{1} M_{1}{ }^{2}\left\{\left[1-\eta_{1}{ }^{2}-\mu^{2}\left(3+\eta_{1}{ }^{2}\right)\right] K_{1} M_{1}{ }^{2}-4 \eta_{1}{ }^{2}\left[2 \mu^{2}\left(1-\mu^{4}\right) \beta_{1}+\left(1-\mu^{2}\right)^{2}\left(1-\beta_{1}\right)\right]\right\}$,

$a_{1}=K_{1}{ }^{2} M_{1}{ }^{4}\left\{\mu^{2} K_{1} M_{1}{ }^{2}+\left(1+\mu^{2}\right)\left(1-\mu^{2}\right)^{2}\left(1-\beta_{1}\right)+2 \mu^{2}\left(1-\mu^{4}\right) \beta_{1}+\eta_{1}{ }^{2}\left(1-\mu^{2}\right)^{2}\left(3-\mu^{2}\right)\left(1-\beta_{1}\right)\right\}$

und

$$
a_{0}=-K_{1}^{3} M_{1}{ }^{6} .
$$

Bevor diese Gleichung 3. Grades näher diskutiert wird, sollen noch einige andere Größen, die von Interesse sind, abgeleitet werden.

d) Geschwindigkeit hinter der Stoßfront

Die $x$-Komponente der Geschwindigkeit der Materie hinter der Front ist nach Gl. (1) gegeben durch

$$
u_{x 2}=v_{x 2}-v_{x 1} .
$$

Nach dem Impulssatz für die $x$-Komponente Gl. $(\mathrm{I}, 52, \mathrm{~b} 1)$ gilt

$$
\begin{aligned}
-\varrho_{1} v_{x 1} u_{x 2}=\varrho_{1} U u_{x 2}= & p_{2}-p_{1} \\
& +\frac{1}{8 \pi}\left\{\left(H_{y 2}\right)^{2}-\left(H_{y 1}\right)^{2}\right\} .
\end{aligned}
$$

Setzt man hier Gl. (38) ein, so bekommt man

$$
\begin{aligned}
\frac{u_{x 2}}{c_{1}} & =\left(1-\mu^{2}\right) M_{1}\left\{1-2\left(1+\mu^{2}\right) \mu^{2} \beta_{1} \frac{1}{K_{1} M_{1}{ }^{2}}\right. \\
& -\left(1-\mu^{2}\right)\left(1-\beta_{1}\right)\left(1-\eta_{1}{ }^{2}\right)\left[\mu^{2}(y C-1)^{2}\right. \\
& \left.\left.+\left(1-\mu^{2}\right) y D(y C+1)-\left(y^{2} C^{2}-1\right)\right] \frac{1}{K_{1} M_{1}{ }^{2}}\right\} .
\end{aligned}
$$

Für die $y$-Komponente der Geschwindigkeit erhält man aus dem Impulssatz

$$
\varrho_{2} v_{x 2} v_{y_{2}}=-\varrho_{1} U u_{y_{2}}=\frac{1}{4 \pi}\left(H_{y_{2}}-H_{y_{1}}\right) H_{x 1} .
$$

Mit Hilfe von Gl. (31) ergibt sich dann

$$
\begin{aligned}
& \frac{u_{y 2}}{c_{1}}=2\left(1-\mu^{2}\right)^{2}\left(1-\beta_{1}\right) \eta_{1} \\
& \cdot+\sqrt{ } 1-\eta_{1}{ }^{2}(1-y C) \frac{1}{K_{1} M_{1}} .
\end{aligned}
$$

e) Verhältnis der materiellen Energiedichte zur Gesamtenergiedichte hinter der Stoßfront

Nach Gl. (23) gilt

$\beta_{2}=\frac{e_{2}}{e_{2}^{*}}=\frac{\left(1-\mu^{2}\right) p_{2}}{\left(1-\mu^{2}\right) p_{2}+\frac{\mu^{2}}{4 \pi}\left(H_{2}\right)^{2}}$.

$$
\begin{aligned}
& \text { Mit Hilfe von Gl. (31) und Gl. (38) wird daraus } \\
& \beta_{2}=\frac{\beta_{1}}{\beta_{1}+\left(1-\beta_{1}\right)\left\{\eta_{1}{ }^{2}+y^{2} C^{2}\left(1-\eta_{1}{ }^{2}\right)\right\} \frac{p_{1}}{p_{2}}}
\end{aligned}
$$

f) Neigung des Magnetfeldes hinter der Front

Das Quadrat des Kosinus des Neigungswinkels vom Magnetfeld auf der Rückseite der Stoßfront ist gegeben durch

$$
\eta_{2}^{2}=\frac{\left(H_{x 2}\right)^{2}}{\left(H_{2}\right)^{2}}=\frac{\eta_{1}^{2}}{\eta_{1}^{2}+y^{2} C^{2}\left(1-\eta_{1}{ }^{2}\right)} .
$$

\section{g) Spezielle Lösung}

Bisher war vorausgesetzt worden nach Gl. (30), daß

$$
\left(v_{x 2}\right)^{2} \neq \frac{1}{4 \pi \varrho_{2}}\left(H_{x 2}\right)^{2}
$$

sei. Es soll nun der Fall

$$
\left(v_{x 2}\right)^{2}=\frac{1}{4 \pi \varrho_{2}}\left(H_{x 2}\right)^{2}
$$

gesondert untersucht werden. Dies ist nach Gl. (29) nur möglich, wenn entweder $H_{y_{1}}=0$ oder

$$
\left(v_{x 1}\right)^{2}=\frac{1}{4 \pi \varrho_{1}}\left(H_{x 1}\right)^{2}=\left(c_{\mathrm{A}_{1}}\right)^{2}
$$

ist. $H_{y 1}=0$ heißt aber Ausbreitung parallel zum Magnetfeld, und dies war schon in Abschn. 2 untersucht worden.

Wir betrachten daher den Fall, daß Gl. (48) und (49) erfüllt ist, aber $H_{y_{1}} \neq 0$ und $H_{y_{2}} \neq 0$. (Wenn $H_{y 2}=0$ sein soll und außerdem Gl. (48) gelten soll, so muß auch $H_{y_{1}}=0$ sein, wie sich leicht zeigen läßt.) Dann ist nach Gl. (31) $C$ zunächst unbestimmt, während die Definition von $D$ nach Gl. (40) nicht mehr sinnvoll ist. Es wird sich aber zeigen, daß der Ausdruck $D(y C+1)$ ebenso wie $C$ endlich bleibt, und nur in dieser Form tritt $D$ in den Gleichungen in Erscheinung.

Aus Gl. (48) und (49) folgt mit Hilfe der Kontinuitätsgleichung, daß $v_{x 1}=v_{x 2}$ ist, und damit be- 
kommt man für diesen Fall $y=\varrho_{2} / \varrho_{1}=1$. Man kann nun $y$ als Funktion von $M_{1}{ }^{2}=x$ an der Stelle

$x_{0}=\left(M_{1}{ }^{2}\right)_{0}=\frac{\left(c_{\mathrm{A}_{1}}\right)^{2}}{c_{1}}=2\left(1-\mu^{2}\right)^{2}\left(1-\beta_{1}\right) \eta_{1}{ }^{2} / K_{1}$

in eine Potenzreihe entwickeln, indem man die Gleichung 3. Grades Gl. (41) für $y$ benutzt. Man erhält dann

$$
y=1+a\left(x-x_{0}\right)+b\left(x-x_{0}\right)^{2}+\ldots,
$$

wobei die Koeffizienten $a$ und $b$ sich ergeben zu

und

$$
a=\frac{K_{1}}{\left(1-\mu^{2}\right)^{2}\left(1-\beta_{1}\right) \eta_{1}^{2}}
$$

$$
\begin{aligned}
b= & \frac{K_{1}{ }^{2}}{2\left(1-\mu^{2}\right)^{6}\left(1-\beta_{1}\right)^{3} \eta_{1}{ }^{4}\left(1-\eta_{1}{ }^{2}\right)} \\
& \cdot\left\{\mu^{2}\left(1+\mu^{2}\right) \beta_{1}-\left(1-\mu^{2}\right)^{2}\left(1-\beta_{1}\right) \eta_{1}{ }^{2}\right. \\
& \left.+\left(1-\mu^{4}\right)\left(1-\beta_{1}\right)\left(1-\eta_{1}{ }^{2}\right)\right\} .
\end{aligned}
$$

Setzt man Gl. (51) in Gl. (32) ein, so wird im Grenzübergang

$$
C=-1 \text { für } x=x_{0} \text { und } y=1
$$

und damit

$$
H_{y 2}=-H_{y 1} \text { für } x=x_{0} \text { und } y=1 .
$$

Weiter ergibt sich im Grenzübergang

$$
D(y C+1)=a x_{0}-\frac{1}{2} b x_{0}^{2} \text { für } x=x_{0} \text { und } y=1 .
$$

Dies in Gl. (38) eingesetzt ergibt

$$
p_{2}=p_{1} \text { für } x=x_{0} \text { und } y=1 .
$$

Weiterhin ist nach Gl. (44)

$$
u_{x 2}=0 \text { für } x=x_{0} \text { und } y=1 .
$$

Dagegen bleibt die Geschwindigkeitskomponente auf der Rückseite der Front in der $y$-Richtung endlich und ist nach Gl. (45):

$$
\begin{gathered}
\frac{u_{y_{2}}}{c_{1}}=2 \sqrt{2}\left(1-\mu^{2}\right)+\sqrt{ } 1-\beta_{1}+\sqrt{ } 1-\eta_{1}^{2} /+V K_{1} \\
\text { für } x=x_{0} \text { und } y=1 .
\end{gathered}
$$

Man hat also bei $x=x_{0}$ die etwas merkwürdige Lösung, daß die Dichte und der Druck auf beiden Seiten der Stoßfront übereinstimmen. Dagegen hat die $y$-Komponente des Magnetfeldes auf der Rückseite der Front das entgegengesetzte Vorzeichen der $y$-Komponente auf der Vorderseite (s. Abb. 4). Der hieraus resultierende Impuls tangential zur Stoßfront wird durch eine Materieströmung mit der Geschwindigkeit $u_{y_{2}}$ aufgenommen. Daß diese Lösung gerade dann möglich ist, wenn die Frontgeschwindigkeit $U$ gleich der Ge- schwindigkeit $c_{\mathbf{A}}$ der Alfvénschen Wellen ist, wird durch folgendes verständlich: Die Fortpflanzungsgeschwindigkeit von Alfvénschen Wellen gilt nicht nur für infinitesimale Störungen, sondern auch für beliebig große $^{8}$. Stellt man sich die Magnetfeldlinien als Saiten vor, die hier aus ihrer Ruhelage ausgelenkt seien, so wird sich deshalb eine solche Auslenkung mit der Geschwindigkeit $c_{\mathrm{A}}$ fortpflanzen.

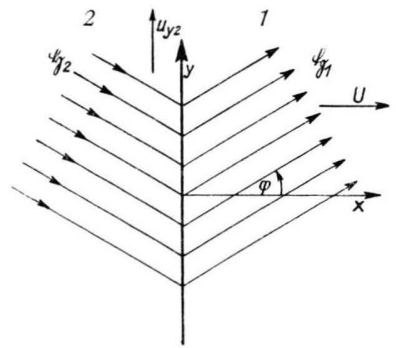

Abb. 4. Stoßfront und Magnetfeld für $U=c_{\mathrm{A} 1}$.

Die Gleichung 3. Grades des Dichteverhältnisses $y$ liefert an der Stelle $x=x_{0}$ noch zwei weitere Lösungen. Für die eine Lösung ist $y>1$ und $H_{y 2}=0$ [d. h. es gilt Gl. (30)], die andere Lösung ergibt $y<1$, was physikalisch nicht sinnvoll ist, da damit eine Entropieerniedrigung verbunden wäre.

\section{Diskussion der Ergebnisse}

a) Es soll nun die Lösung der Gleichung 3. Grades, Gl. (41), für das Dichteverhältnis $y=\varrho_{2} / \varrho_{1}$ diskutiert werden.

Keinen Dichtesprung wird man dann bekommen, wenn die Geschwindigkeit der Stoßfront gleich der Schallgeschwindigkeit vor der Front ist. Die Lösung $y=1$ bekommt man also, wenn

und wenn

$$
U=c_{+1} \text { d. h. } M_{+1}=\frac{U}{c_{+1}}=1
$$

$$
U=c_{-1} \text { d. h. } M_{-1}=\frac{U}{c_{-1}}=1
$$

ist. Außerdem hat sich in Abschn. $4 \mathrm{~g}$ herausgestellt, daß $y=1$ eine Lösung von Gl. (41) ist, wenn

$$
U=c_{\mathrm{A}_{1}} \text { d. h. } M_{\mathrm{A}_{1}}=\frac{U}{c_{\mathrm{A}_{1}}}=1
$$

ist. Es gilt nun stets für die beiden Schallgeschwindigkeiten $c_{+}$und $c_{-}$sowie für die Geschwindigkeit der Alfvénschen Wellen

$$
c_{-}<c_{\mathrm{A}}<c_{+} \text {, }
$$

${ }^{8}$ H. Alfvén, Cosmical Electrodynamics, p. 86 Oxford 1950 . 


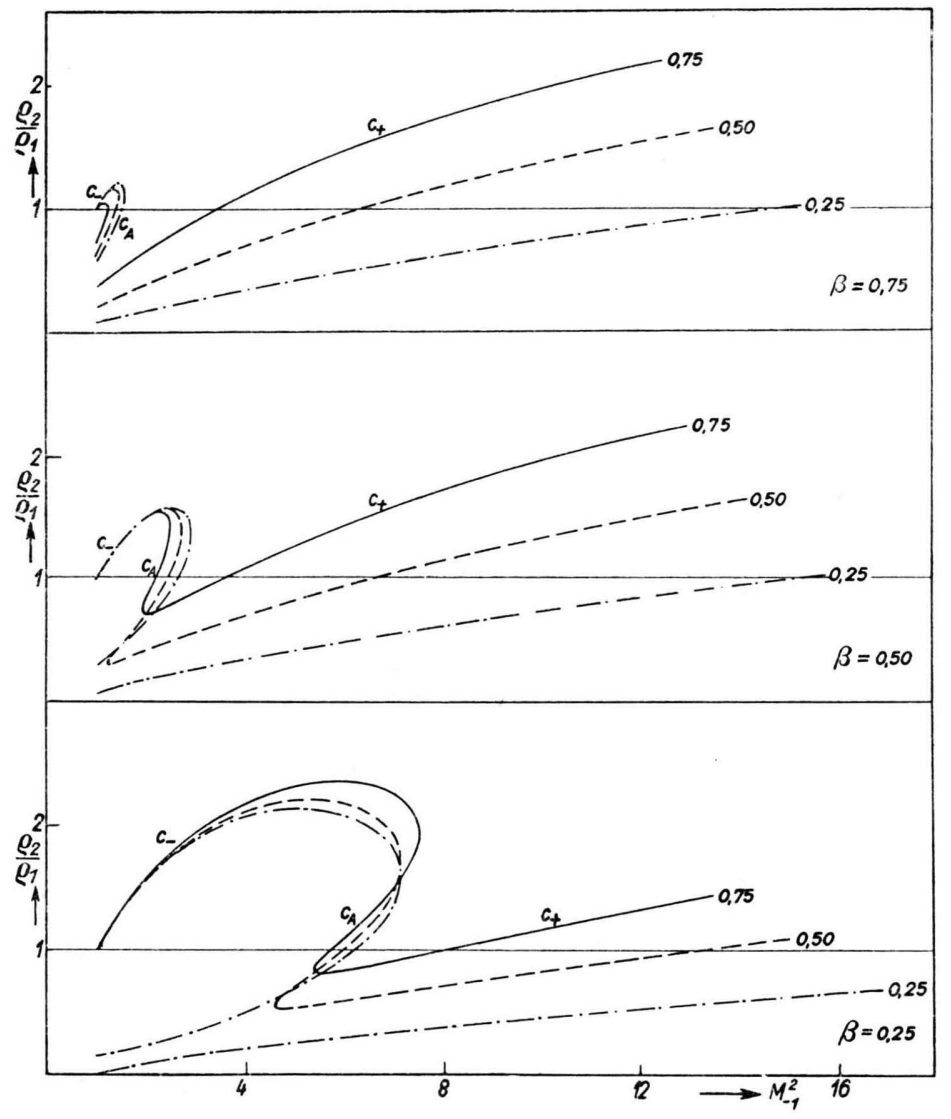

Abb. 5. Dichteverhältnis $y=\varrho_{2} / \varrho_{1}$ in Abhängigkeit von $M_{-1}^{2}$ für $\beta_{1}=0,75 ; 0,5$ und 0,25 . Der Kurvenparameter ist jeweils $\eta_{1}^{2}=\cos ^{2} \varphi_{1}=0,75 ; 0,5$ und 0,25 .

wobei $c_{+}$und $c_{-}$nach Gl. (27) gegeben sind, während man für $c_{\mathrm{A}}$ erhält:

$$
c_{\mathrm{A}}^{2}=\frac{1-\mu^{2}}{\mu^{2}} \cdot \frac{1-\beta}{\beta} \eta^{2} \frac{p}{\varrho} .
$$

Löst man daher Gl. (41) auf diese Weise, daß man die Machsche Zahl $M$ mit der kleinsten Schallgeschwindigkeit $c_{-}$definiert, so bekommt man für $M_{-1}=1$ die Lösung $y=1$. Weiterhin erhält man wieder die Lösung $y=1$, wenn $M_{-1}=c_{\mathrm{A}_{1}} / c_{-1}(>1)$ ist, und schließlich ergibt sich die Lösung $y=1$, wenn $M_{-1}=c_{+1} / c_{-1}(>1)$ erreicht ist.

Zur genaueren Untersuchung wurde die Gl. (41) als Funktion von $M^{2}{ }_{-1}$ für $\gamma=5 / 3$ (einatomiges Gas) und für verschiedene $\beta_{1}$ und $\eta_{1}$ numerisch gelöst. Weiterhin wurden das Druckverhältnis, die Geschwindigkeiten hinter der Front, die Richtungsänderung des Magnetfeldes und das Verhältnis der inneren Energie des Gases zur gesamten inneren Energie numerisch bestimmt. Die ersten
Rechnungen erfolgten mit Hilfe der Göttinger elektronischen Rechenmaschine G 1, später konnte die große elektronische Rechenmaschine G 2 benutzt werden ${ }^{9}$. Die Resultate sind in den Abb. 5 und 6 als Funktion von $M^{2}{ }_{-1}$ für verschiedene Parameterwerte $\beta_{1}$ und $\eta_{1}^{2}$ aufgetragen.

Das Dichteverhältnis y (s. Abb. 5) nimmt mit wachsendem $M^{2}{ }_{-1}$ zunächst zu. Von einem bestimmten Wert von $M^{2}{ }_{-1}$ (der auch schon bei $M^{2}{ }_{-1} \leq 1$ liegen kann) sind drei Lösungen für $y$ möglich, von denen aber zunächst $y_{2}$ und $y_{3}$ kleiner als 1 sind. Diese Lösungen sind physikalisch nicht sinnvoll, da sie mit einer Entropieabnahme verbunden wären. Erst bei $M^{2}{ }_{-1}=c_{\mathrm{A}}{ }^{2} / c^{2}{ }_{-1}$ wird $y_{2}=1$. Mit wachsendem $M^{2}{ }_{-1}$ nimmt dann $y_{1}$ ab und $y_{2} \mathrm{zu}$, bis man an einen Punkt gelangt, an dem $y_{1}=y_{2}$ ist, während $y_{3}<1$ ist. Von da ab existiert nur noch die Lösung $y_{3}$, die für die Stelle

$$
M_{-1}^{2}=c^{2}{ }_{+1} / c^{2}{ }_{-1}
$$

den Wert 1 erreicht. Das Dichteverhältnis $y$ nimmt dann mit wachsender Machzahl stets zu, um für sehr große Machzahlen den Grenzwert 4 wie bei den normalen hydrodynamischen Stoß-

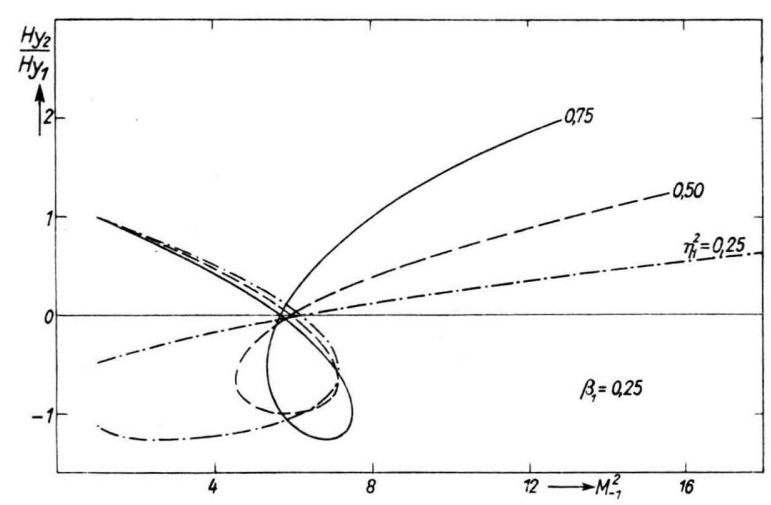

Abb.6. Richtungsänderung des Magnetfeldes $\left(H_{y_{2}} / H_{y_{1}}\right)$ in Abhängigkeit von $M_{-1}^{2}$ für $\beta_{1}=0,25$ sowie für $\eta_{1}^{2}=0,75 ; 0,5 ; 0,25$.

${ }^{9}$ H. Oehlmann, Vorträge über Rechenanlagen (herausgegeben von L. Biermann) S. 60, Göttingen 1953. 
wellen zu erreichen. Die Erklärung hierfür ist die gleiche wie bei den senkrechten Stoßwellen (s. Abschn. 3). Auch bei sehr großen Machschen Zahlen können bei bestimmten Parameterwerten $\beta_{1}$

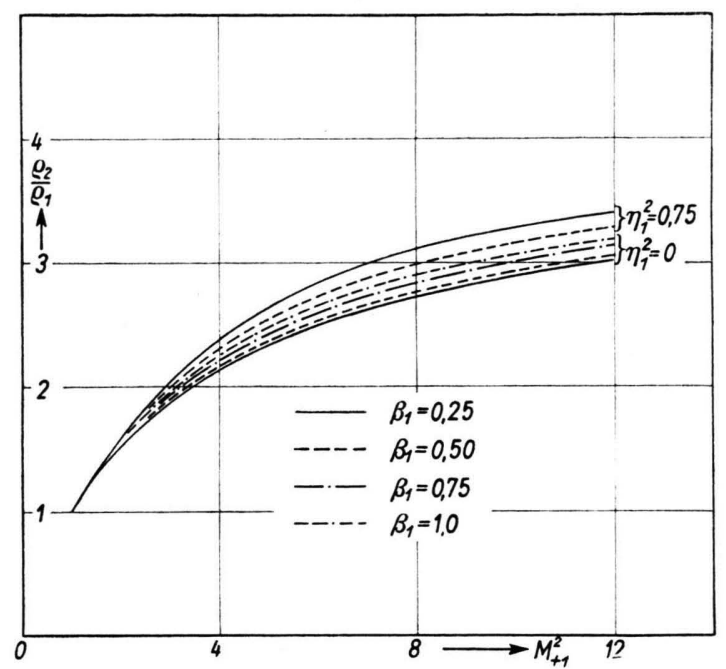

Abb. 7. Dichteverhältnis $y=\varrho_{2} / \varrho_{1}$ in Abhängigkeit von $M_{+1}^{2}$ für $\beta_{1}=1,0 ; 0,75 ; 0,5$ und 0,25 sowie für $\eta_{1}{ }^{2}=0$ und 0,75 .

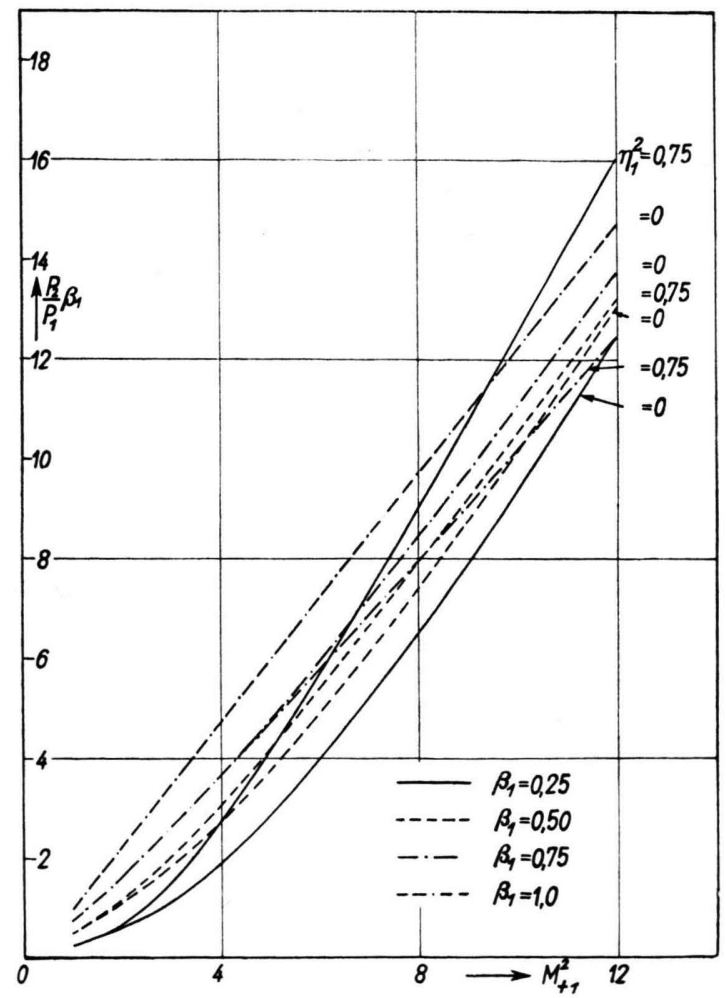

Abb. 8. Druckverhältnis $p_{2} / p_{1} \cdot \beta_{1}$ in Abhängigkeit von $M_{+1}^{2}$ für $\beta_{1}=1,0 ; 0,75 ; 0,5$ und 0,25 sowie für $\eta_{1}{ }^{2}=0$ und 0,75 . und $\eta_{1} \mathrm{u}$. U. wieder drei reelle Lösungen existieren, jedoch sind die zwei zusätzlichen Lösungen negativ und daher nicht sinnvoll.

Man hat also drei verschiedene Arten von stationären Stoßwellen, die im Grenzübergang zu unendlich schwachen Stößen in Wellen mit der Geschwindigkeit $c_{-}, c_{\mathrm{A}}$ und $c_{+}$übergehen. Die Rechnungen zeigen, daß die Stoßwellen, die zu der Schallgeschwindigkeit $c_{+}$gehören, für jede Machsche Zahl $M_{+1}>1$ existieren können. Dagegen sind die beiden anderen Arten von Stoßwellen nur in einem bestimmten Bereich $1<M_{-1} \leq a$ bzw.

$$
c_{\mathrm{A} 1} / c_{-1}<M_{-1} \leq a
$$

möglich. Für große Machsche Zahlen können diese nicht auftreten, außerdem können sie nur in einem um so kleineren Bereich von $M_{-1}$ existieren, je schwächer das Magnetfeld $\left(\beta_{1} \rightarrow 1\right)$ ist.

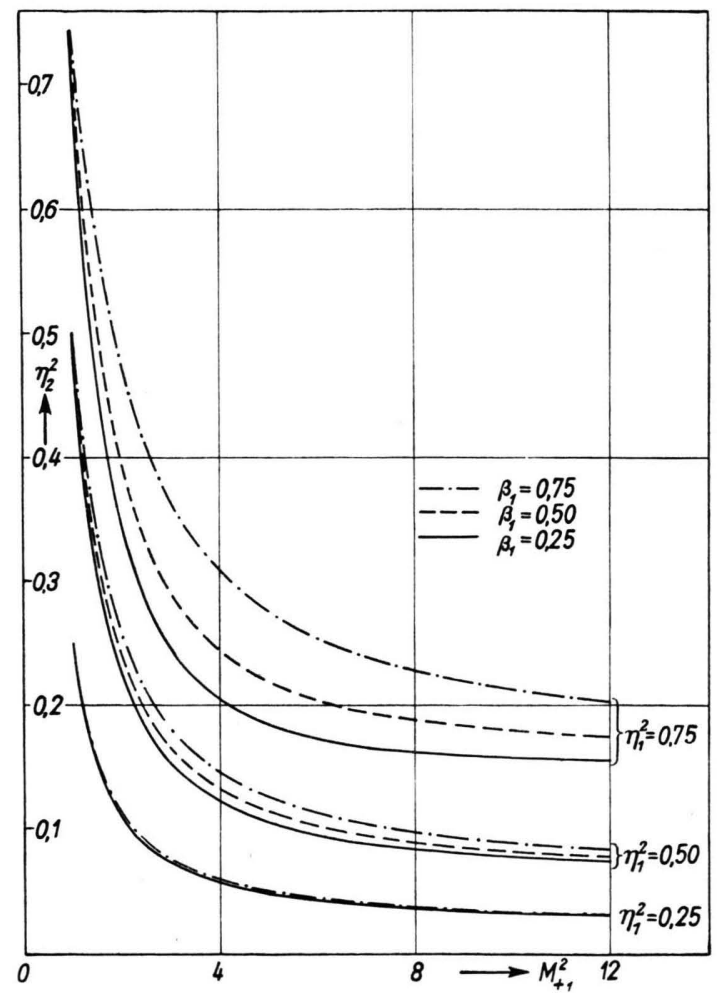

Abb. 9. Richtungsänderung des Magnetfeldes $\left(\eta_{2}{ }^{2}\right)$ in Abhängigkeit von $M_{+1}{ }^{2}$ für $\beta_{1}=0,75 ; 0,5 ; 0,25$ sowie für $\eta_{1}^{2}=0,75 ; 0,5 ; 0,25$.

Im Fall $\eta_{1}=0$ (senkrechte Stoßwellen) wird aus Gl. (41) eine quadratische Gleichung, von der nur eine Lösung physikalisch sinnvoll ist, da die andere Lösung negative Dichteverhältnisse ergibt. 
Hier hat man also stets nur eine Art von Stoßwellen, die für $M_{+_{1}}=U / c_{+1}=U / c_{1}{ }^{*} \rightarrow 1$ in Schallwellen mit der Geschwindigkeit $c_{1} *$ übergehen (s. Abschn. 3). In Abb. 7 ist das Dichteverhältnis und in Abb. 8 das Druckverhältnis für $\eta_{1}=0$ bei verschiedenen Werten von $\beta_{1}$ zusammen mit noch anderen Parameterwerten $\eta_{1}$ als Funktion von $M^{2}{ }_{+1}=U^{2} / c^{2}{ }_{+1}$ aufgetragen.

b) Die Richtungsänderung des Magnetfeldes hinter der Stoßfront für die $c_{-}$-Wellen ist so, daß die $y$-Komponente des Magnetfeldes mit wachsen$\operatorname{dem} M_{-1}$ zunächst abnimmt. Dies ist anschaulich so zu verstehen, daß die magnetische Energie auf diese Weise erniedrigt wird. Wenn $M_{-1}=c_{\mathrm{A}} / c_{-1}$ erreicht ist, steht das Magnetfeld senkrecht auf der Front $\left(H_{y 2}=0\right.$, s. Abschn. 4). Dann nimmt die $y$ Komponente schließlich in anderer Richtung wieder zu, während die $y$-Komponente des Magnetfeldes bei den $c_{\mathrm{A}}$-Wellen abnimmt, bis sie mit der der $c_{-}$-Wellen übereinstimmt (s. Abb. 6). Bei den $c_{+}$-Wellen nimmt die $y$-Komponente des Magnetfeldes stets zu, das Magnetfeld wird durch die Stoßfront umgeschmissen (s. Abb. 6 und Abb. 9). Die magnetische Energie wächst zwar auf diese Weise stets, aber da die innere Energie des Gases für große Machsche Zahlen viel stärker ansteigt und die Zunahme der magnetischen Energie begrenzt bleibt, nimmt schließlich das Verhältnis der magnetischen Energie zur Gesamtenergie für große Machsche Zahlen doch ab. Für kleine Machsche Zahlen kann jedoch bei bestimmten Werten von $\beta_{1}$ und $\eta_{1}$ dieses Verhältnis auch zunehmen (s. Abb. 10).

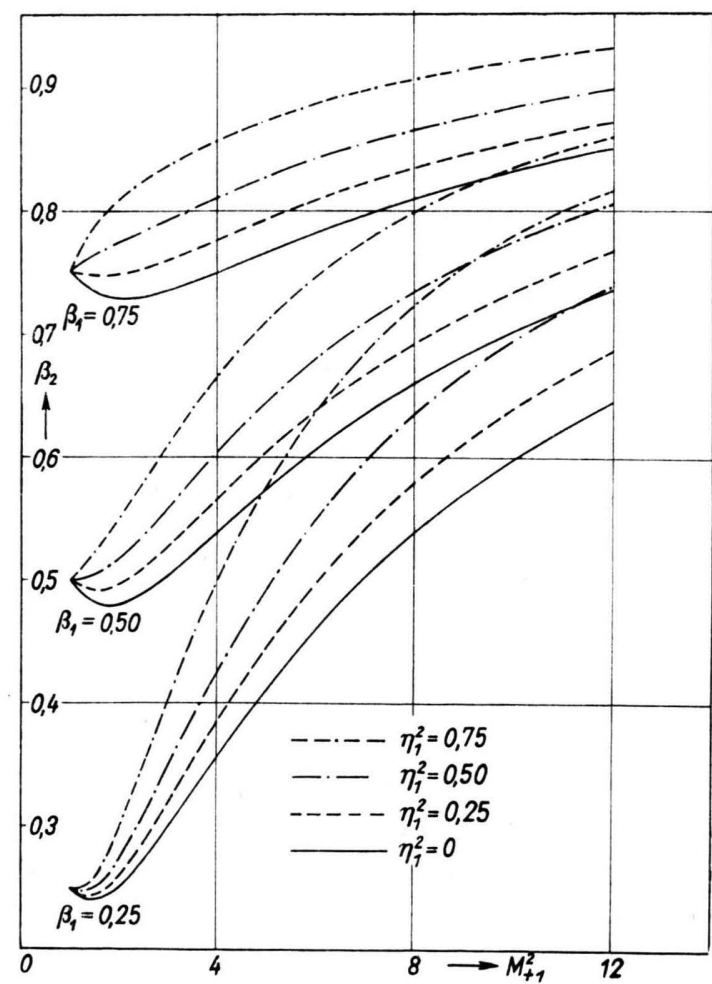

Abb. 10. Verhältnis $\beta_{2}$ der inneren Energie $e_{2}$ des Gases zur gesamten inneren Energie $e_{2}{ }^{*}$ hinter der Stoßfront in Abhängigkeit von $M_{+1}^{2}$ für $\beta_{1}=0,75,0,5 ; 0,25$ sowie für $\eta_{1}^{2}=0,75 ; 0,5 ; 0,25$ und 0 .

Herrn Professor Biermann möchte ich vielmals für fördernde Diskussionen und sein Interesse an der Arbeit danken, ebenso Fräulein Trefftz für zahlreiche Ratschläge und Herrn Oehlmann für die Hilfe bei den Rechnungen auf $\operatorname{der}$ G 2 . 